Revista Arbitrada Interdisciplinaria KOINONIA

Año 2020 . Vol V. $\mathrm{N}^{\circ} 1$. Especial Educación

Hecho el depósito de Ley: FA2016000010 ISSN: 2542-3088

FUNDACIÓN KOINONIA (F.K). Santa Ana de Coro. Venezuela.

Cristian Javier Luna-Miranda; Darwin Gabriel García-Herrera; Ana Zulema Castro-Salazar; Juan Carlos Erazo-Álvarez

http://dx.doi.org/10.35381/r.k.v5i1.806

\title{
Uso alternativo de las TIC en Educación Básica Elemental para desarrollar la lectoescritura
}

\section{Alternative use of ICT in Basic Elementary Education to develop literacy}

Cristian Javier Luna-Miranda

cristian.luna@psg.ucacue.edu.ec

Universidad Católica de Cuenca, Azogues

Ecuador

https://orcid.org/0000-0002-3314-2449

Darwin Gabriel García-Herrera

dggarciah@ucacue.edu.ec

Universidad Católica de Cuenca, Azogues

Ecuador

https://orcid.org/0000-0001-6813-8100

Ana Zulema Castro-Salazar

azcastros@ucacue.edu.ec

Universidad Católica de Cuenca, Azogues

Ecuador

https://orcid.org/0000-0002-3837-314X

Juan Carlos Erazo-Álvarez

jcerazo@ucacue.edu.ec

Universidad Católica de Cuenca, Cuenca

Ecuador

https://orcid.org/0000-0001-6480-2270

Recibido: 25 de abril de 2020

Revisado: 30 de mayo de 2020

Aprobado: 15 de junio de 2020

Publicado: 30 de junio de 2020 


\title{
RESUMEN
}

La presente investigación tiene como objetivo determinar los niveles de aceptación e implementación de las Tecnologías de la Información y Comunicación (TIC) en el proceso enseñanza a la lectoescritura en el subnivel de básica elemental. El trabajo investigativo fue de tipo descriptivo. Los resultados demuestran que los docentes consideran importante el uso de las TIC para el desarrollo de la lectoescritura, puesto que los niños muestran altos índices de interés ante el uso de tecnología, con esta premisa se establece una propuesta de implementación de las TIC para el desarrollo de lectoescritura en la Escuela, misma que motive a los estudiantes hacia una equilibrada e interpretativa Educación, basada en la adquisición de competencias, habilidades y el desarrollo de la imaginación, plasmadas en textos enriquecidos con el uso de herramientas tecnológicas, que incrementen la participación individual y colectiva dentro y fuera de las instituciones educativas.

Descriptores: Tecnología de la información; informática educativa, promoción de la lectura; orientación para la lectura. (Palabras tomadas del Tesauro UNESCO).

\begin{abstract}
This research aims to determine the levels of acceptance and implementation of Information and Communication Technologies (ICT) in the teaching process of literacy at the elementary basic sub-level. The research work was descriptive. The results show that teachers consider the use of ICTs to be important for the development of literacy, since children show high levels of interest in the use of technology. With this premise, a proposal for the implementation of ICTs for development is established. of literacy in the School, which motivates students towards a balanced and interpretive Education, based on the acquisition of competences, skills and the development of the imagination, embodied in texts enriched with the use of technological tools, which increase individual participation and collective inside and outside educational institutions.
\end{abstract}

Descriptors: Information technology; computer uses in education; reading promotion; reading guidance. (Words taken from the UNESCO Thesaurus). 


\section{INTRODUCCIÓN}

En la actualidad Las Tecnologías de la Información y Comunicación [TIC] son herramientas de apoyo pedagógico, las cuales brindan un aprovechamiento notorio dentro del aula de clase permitiéndole a los estudiantes demostrar habilidades y cualidades ahondadas y dándolas a flote dentro de la misma muestra una gestión y transformación de información, es decir se les permite experimentar el uso de programas, aplicaciones y ordenadores: crear, almacenar, modificar, recuperar y proteger información, (Tangarife, Blanco, \& Diaz, 2016) manifiesta que las TIC generan grandes cambios en la sociedad; mismas que puestas en práctica generan descontento en los migrantes digitales por el temor aceptar y manipular la tecnología (Vélez-Loor, et al., 2020).

Por otro lado los nativos digitales les motiva el manejo y la utilización, mismos que nacen con el chip de tecnología aprenden más haciendo, practicando, manipulando que escuchando, esta situación de los avances tecnológicos no son afanes, son realidades que se presentan en la actualidad por cuanto la vida y progresos diarios está programada a base de tecnología, esto permite sacar provecho de las oportunidades que presenta la vida observando el lado positivo de las cosas como es optimación de tiempo y lugar, los constantes cambios e innovaciones tecnológicas, a través del internet, facilita documentos, herramientas, programas, datos, que pueden ser compartidos con todo el mundo de forma rápida y eficiente.

Los beneficiarios son todas las personas que requieren de información de ahí parte la cultura global que se encuentra inmersa la sociedad del conocimiento tecnológico e innovador con el fin de adaptarse al dinamismo del entorno al que pertenece y comparte su vida diaria (Andersen -Boris Branisa, \& Canelas 2016). Las herramientas eficientes en la tecnología permite adaptar a los niños, niñas y jóvenes a la sociedad evolutiva que está figurando a base de tecnología, permitiendo desarrollar habilidades para la vida con el manejo de información y comunicación con diferentes personas y de diferentes lugares del mundo (Coll, 2008). 
Por este sentido insertar las TIC en el campo educativo, hace posible la conexión con el mundo exterior aprovechando las ventajas que tienen estas herramientas al ser utilizadas y compartidas desde cualquier lugar y tiempo, interactuando con personas de culturas diversas mediante juegos interactivos, espacios sociales, producción y ejecución de contenidos, audiolibros, recursos educativos del internet (Chacha-Supe \& Rosero-Morales, 2020).

(Luz, 2018) alega que la incursión de las TIC mediadoras en lectoescritura, surge debido a los grandes cambios que ha generado la globalización, ahí nace la necesidad de estímulo de la tecnología en educación. En lectoescritura hay herramientas que dinamizan el tiempo en los docentes haciendo de esto un aprendizaje motivador e interesante para que el alumno aprenda a su ritmo e integre a su necesidad de aprender a en cualquier tiempo y espacio, el estudiante no está sometido a un solo tipo de aprendizaje, ello puede hacer de acuerdo a su necesidad y ambiente donde se desarrolla, cada uno tiene su estilo, esto ha generado la implementación de la tecnología en educación el gusto por lo que hacen, mas no lo que el maestro impone. Las ventajas de la participación activa de herramientas es la variedad en contenido.

La TIC en instituciones educativas generaliza el conocimiento, si puede ser un restrictivo para unos al no tener acceso a la tecnología, pero ahí la importancia del docente, incluir en las clases, ahí el alumno se vuelve más activo porque sabe el tiempo que debe aprovechar a la tecnología, el docente debe buscar herramientas o aplicaciones necesarias para que la clase impartida sea de calidad, lo cual posibilita formarse en la lectura como esencia educativa (Borregales, 2020).

Con estas premisas la investigación tiene como objetivo determinar los niveles de aceptación e implementación de las Tecnologías de la Información y Comunicación (TIC) en el proceso enseñanza a la lectoescritura en el subnivel de básica elemental. 


\section{Referencial teórico}

\section{Las TIC en educación}

(Suárez et al., 2015) afirma que la importancia de las TIC en los escenarios educativos y en la sociedad es abrir puertas a la innovación, en este ámbito busca mejorar el proceso de enseñanza aprendizaje, propiciar incorporar recursos y herramientas tecnológicas para mejorar las competencias y destrezas que estas requieren en diferentes disciplinas del conocimiento. Todo proceso innovador requiere de la participación activa de sus integrantes involucrados en este caso docentes, estudiantes para lo cual es necesario acordar mejoras en beneficio de los educandos, quien son el foco de atracción educativa, pero también se requiere la intervención de los padres de familia que provean los equipos tecnológicos básicos para dar una solución práctica y mejorar las destrezas del docente con respuesta a una necesidad y generar motores de cambio (Bazán-Ramírez et al., 2020). (Palos Toscano et al., 2017) así mismo un modelo de gestión de aprendizaje se hace posible mediante la ejecución de proyecto que se traza como institución, tomando decisiones adecuadas a la necesidad generando ideas, desarrollando habilidades complementarias que involucran el avance de competencias lectoras para construir un aprendizaje que satisfaga a la sociedad.

\section{Lectoescritura}

(Prado-Huante, 1949) se le conoce a la lectoescritura como una relación entre el texto y lo subjetivo, capacidad de escuchar, leer, escribir, hablar con una intensión de propósito y que conlleva destrezas para enlazar la expectativa del docente con el resultado obtenido en el proceso de la lectoescritura en conjunto con la tecnología para un mejor aprovechamiento en la enseñanza complementaria como su fácil atractivo para el estudiante y así se tornaría más divertida y dinámica la hora de preparar y construir una clase, con ello despierta el interés en usar herramientas aplicables a la jornada pedagógica y sumar expectativas y temáticas de estudio desarrollando un pensamiento más crítico y constructivo para resolver conflictos propios y escuchar opiniones ajenas 
acorde a una solución oportuna (Dovale, 2018).

(Palos Toscano et al., 2017) desde la etapa inicial es de suma importancia el reconocer la estructura de los textos para una revisión, planeación, ejecución en donde el docente crea ejercicios con herramientas de la web tomando como mecanismo apropiado métodos y destrezas para los niños del nuevo milenio. El adquirir conocimientos significativos y reflexivos ejecutando estas herramientas, el maestro brinda una buena estructura y vínculos que fortalezcan el proceso de aprendizaje que pueden ser utilizadas en varias áreas de competencia adquiridas por los estudiantes.

De las cuales abrirán camino a competencias y así lograr algo productivo y dar paso a una aptitud de elegibilidad según su conveniencia a la escritura y lectura y estas serán entrelazadas con el desarrollo del niño y el enseñares a leer y escribir es un reto en los grados elementales de todas las instituciones educativas (Toro \& Cervera, 2015). Haciendo referencia a la inclusión de herramientas tecnológicas en el contexto educativo el juego es un recurso natural que es nato en todo ser humano y con la personalización se pretende que el estudiante se convierta en un ser activo tecnológicamente hablando mas no un migrante tecnológico.

(Duart, 2012) de acuerdo a los aspectos tecnológicos es necesario el uso de software y conectividad estableciendo una relación entre estas partes para su funcionalidad, para lo cual el maestro o maestra debe estar actualizándose periódicamente, por cuanto la tecnología siempre traen un constante cambio, y los padres también son responsables de fijarse en su uso en los hogares porque siempre al estudiante le despierta el interés de curiosidad ahí donde la información se filtra por programas no apto para menores de edad, los estudiantes también deben colaborar entre ellos y de esta manera aquietar un aprendizaje significativo, que determine su uso.

Al mismo tiempo permite el trabajo en equipo, la autoconfianza, respeto, solidaridad y disponibilidad al momento de trabajar, siendo el mismo estudiante autosuficiente para afrontar diferentes situaciones de la vida cotidiana. La sensibilización en las familias sobre uso de las TIC en el proceso de lectoescritura ha ganado ventaja 
involucrando a este proceso a padres, madres de familia y docentes desde sus hogares y dotando de la herramienta tecnológica que aporten a esta iniciativa, siempre pensando primero en beneficio de la niñez y adolescencia, creando en ellos el espíritu de creatividad, motivación y gusto por la lectura y escritura desde sus bases iniciales.

De esta manera sentir gusto por lo que realizan en toda su vida escolar y formar seres competentes y críticos que beneficiaran a la nación, ahí los llamados intermediarios de la educación docentes proveer las diferentes herramientas de acuerdo como se desarrolle las destrezas sabiendo que para cada una hay diferentes aplicaciones y revisar la que está más acorde a la realidad de su entorno (Tangarife, Blanco, \& Diaz, 2016).

(Luz, 2018) comprender la relación entre las TIC y el uso del mismo son dos procesos que van de la mano para una representación intelectual y que requieren de un Proceso de pensamiento sujeta a una interacción y construcción de un producto final, a una experiencia previa como lector y escritor al contexto. La lectura representa una interacción del sujeto con el producto y este a una experiencia significativa a partir de un sistema innovador que de significado al conocimiento y habilidades

(Bazán-Ramírez et al., 2020) consideran que los fonemas es la mínima unidad distintiva de la lenguaje y que se descubre durante el aprendizaje de la lectoescritura. Lo que involucra una dirección múltiple Entre los criterios para decidir qué constituye o no un fonema. Para ello se requiere que exista una función distintiva: son sonidos del habla que permiten distinguir palabras en una lengua.

Desde un punto de vista estructural, el fonema pertenece a la lengua, mientras que el sonido pertenece al habla, dada la distinción entre fonema y fono, existe otra forma de concebir un fonema como una especificación incompleta de rasgos fonéticos. Esta relación es de hecho equivalente a la del fonema como conjunto de fonos: el fonema sería el conjunto de rasgos fonéticos comunes a todos los fonos que forman la clase de equivalencia del fonema.

(Escotto, 2014) refiere que los problemas de la lectura se muestras de maneras sorpresivas y en varios ámbitos. Varios casos presentan un comportamiento inadecuado 
y desinterés en el aula porque de cierta manera se sienten incomodos.

Los estudiantes que tienen dificultades con la lectura podrían evitar por completo la interacción de leer y transcribir el mismo. Esto puede ocurrir en el hogar o en la institución educativa. El maestro puede notar un bajo rendimiento y problemas específicos con la lectura. Es posible que no sepa cómo pronunciar ciertas palabras. o, quizá no pueda explicar de qué se trataba una texto o frase.(Bajaña Delgado, 2017).

La investigación tiene como interés primordial mejorar la lectoescritura por medio de técnicas activas y así superar el índice bajo de rendimiento en el área planteada de estudio y ello permita organizar de mejor manera los conocimientos para desenvolverse ante situaciones cotidianas.

(Escotto, 2014) expone que el desconocimiento de técnicas renovadas de lectura y escritura conlleva a que los niños y niñas no tengan un ilustración significativa y esto arrastre y perjudica al momento de reflejar una óptima lectura y escritura, trayendo como consecuencia un déficit de rendimiento en el aula por lo que se vuelve rotundamente agotador en función de lo que el nuño va a realizar con relación a leer y escribir.

(Tamayo Lorenzo, 2017) indaga que la relación entre la lectura con el conocimiento facilita totalmente que los niños entiendan lo que leen y se les posibilite crear sus observaciones y conclusiones de lo relevante de la lectura.

(Bajaña Delgado, 2017) define que las acciones tomadas dentro del hogar para anticipar una lectura deben ser óptimas y cristalizadas a partir del vínculo familiar llevando esto consigo aspectos positivos de parte del niño llevándole esto a la motivación hacia la lectoescritura.

(Porta \& Anglat, 2011) expone que en la actualidad los resultados pedagógicos se convierten en un fundamento de desarrollo hacia las competencias de lectura y escritura. (Palos Toscano et al., 2017) aseveran que leer es muy importante para el proceso de aprendizaje, es ahí en donde el estudiante desarrolla muchas habilidades y capacidades que los hace aptos para cualquier aspecto académico; mientras más dedicación tiene hacia la lectura mejor será su léxico y ortografía, así como también su capacidad de escribir textos académicos. 
La educación no solo se basa en las enseñanzas impartidas por el docente, sino también de las metodologías que se usa para llegar al aprendizaje verdadero, consciente y eficaz; es aquí en donde el docente usa varias técnicas según su conveniencia para lograr el objetivo según el currículo.

(Magán-Hervás \& Gértrudix-Barrio, 2017) apuntan que el uso de audiovisuales es una gran disyuntiva como metodología para la lectoescritura, pues los niños son altamente activos y mediante esta técnica podrán escuchar y conjuntamente comparar cada palabra o silaba según su fonología y composición.

(Navarro et al., 2016) mencionan que el proceso de lectura se basa en el acto de comprensión de los textos, no sólo lo que dice explícitamente, sino también de lo que el autor trata de comunicar, su mensaje implícito. (Tangarife, Blanco, \& Diaz, 2016) alegan que la epistemología aporta mucho para el estudio de la lectura, así como también para la formación de los lectores, haciéndolos más capacitados para asimilar y valorar sus capacitadades lectoras.

Las metodologías de antaño han sido un arma en contra de la lectura, pues bien animaba a una lectura mecánica en donde mientras más palabras lea el estudiante es de más provecho, no capacitaba al mismo a entender lo dicho en el texto, y el dicente no era idóneo para desglosar la intención del autor.

Con los años las personas se han olvidado casi por completo el ámbito lector, tomando el protagonismo las imágenes, lo electrónico y las redes sociales, a los niños y jóvenes ya no les gusta leer, por lo tanto es muy complicado comprender un texto. (Navarro et al., 2016) indican que los filósofos incorporan sus propias estrategias y métodos para la comprensión de la lectura, ellos consideran a ésta práctica muy relevante para el progreso académico y social de las personas.

Indagar, aplicar e implementar el uso de las TIC para mejorar la lectoescritura en niños de básica elemental de la Escuela de Educación General Básica Emilio Abad Aguilar, y mejorar el proceso académico eficaz de dominio y comprensión de la lectura, en base a una experiencia significativa poco tradicional. 


\section{MÉTODO}

Para el desarrollo de la presente investigación se realizó una investigación descriptiva, no experimental de cohorte transversal (Argudo-Tello, Erazo-Álvarez, \& Narváez-Zurita, 2019), la técnica para recolectar los datos fue la encuesta que contienen 8 preguntas con relación a la falta de resultado de aprendizajes en lectoescritura y el bajo uso de recursos tecnológicos, tales preguntas fueron validadas por el Alfa de Cronbach dando como resultado a 0,743 de 8 preguntas elaboradas y examinadas por el mismo (Lazo-Torres, Erazo-Álvarez, \& Narváez-Zurita, 2019). La muestra empleada es de 26 docentes de las jornadas Matutina y Vespertina de Educación Básica Elemental de la Escuela Emilio Abad. Los datos obtenidos serán analizados a través de pruebas estadísticas y la ayuda del software SPSS (Mendieta-Ortega, Erazo-Álvarez, \& Narváez-Zurita, 2020).

\section{RESULTADOS}

A continuación, se muestra los resultados obtenidos de las encuestas realizadas a los 26 docentes de educación básica elemental de la escuela Emilio Abad:

\section{Tabla 1}

Le parece positivo el ir integrando progresivamente las TIC en su jornada laboral

\begin{tabular}{lccccc} 
& & Frecuencia & Porcentaje & Porcentaje válido & $\begin{array}{c}\text { Porcentaje } \\
\text { acumulado }\end{array}$ \\
\hline \multirow{3}{*}{ Válidos } & Mucho & 19 & 70,4 & 73,1 & 73,1 \\
& Poco & 4 & 14,8 & 15,4 & 88,5 \\
\multirow{3}{*}{ Perdidos } & Casi nada & 3 & 11,1 & 11,5 & 100,0 \\
& Total & 26 & 96,3 & 100,0 & \\
& Sistema & 1 & 3,7 & & \\
\hline
\end{tabular}

Fuente: Elaboración propia.

Los docentes antes mencionados indican que el 70,4\% respondieron que la propuesta da como positivo que las TIC mejora el proceso de enseñanza aprendizaje en la jornada laboral dejando $3,7 \%$ a un resultado negativo 
Revista Arbitrada Interdisciplinaria KOINONIA

Año 2020 . Vol V. $\mathrm{N}^{\circ} 1$. Especial Educación

Hecho el depósito de Ley: FA2016000010

ISSN: 2542-3088

FUNDACIÓN KOINONIA (F.K). Santa Ana de Coro. Venezuela.

Cristian Javier Luna-Miranda; Darwin Gabriel García-Herrera; Ana Zulema Castro-Salazar; Juan Carlos Erazo-Álvarez

\section{Tabla 2}

Las TIC favorecen al aprendizaje significativo en los alumnos.

\begin{tabular}{lccccc} 
& & Frecuencia & Porcentaje & Porcentaje válido & $\begin{array}{c}\text { Porcentaje } \\
\text { acumulado }\end{array}$ \\
\hline \multirow{4}{*}{ Válidos } & Mucho & 19 & 70,4 & 73,1 & 73,1 \\
& Poco & 5 & 18,5 & 19,2 & 92,3 \\
\multirow{3}{*}{ Perdidos } & Nada & 2 & 7,4 & 7,7 & 100,0 \\
& Total & 26 & 96,3 & 100,0 & \\
& Total & 1 & 3,7 & & \\
\hline
\end{tabular}

Fuente: Elaboración propia.

En la encuesta a los docentes, el 70,4\% de docentes reflejan que un desempeño de aprendizaje favorece a la comprensión autónoma significativa, y el 7,4\% a un rendimiento bajo, únicamente el $18,5 \%$ presentan poco rendimiento en el proceso escolar de sus estudiantes.

\section{Tabla 3}

Considera introducir a las TIC en sus clases para un aprendizaje favorecedor en sus alumnos.

\begin{tabular}{|c|c|c|c|c|c|}
\hline & & Frecuencia & Porcentaje & Porcentaje válido & $\begin{array}{l}\text { Porcentaje } \\
\text { acumulado }\end{array}$ \\
\hline & $\mathrm{Si}$ & 21 & 77,8 & 80,8 & 80,8 \\
\hline \multirow[t]{2}{*}{ Válidos } & No & 5 & 18,5 & 19,2 & 100,0 \\
\hline & Total & 26 & 96,3 & 100,0 & \\
\hline Perdidos & Sistema & 1 & 3,7 & & \\
\hline \multicolumn{2}{|c|}{ Total } & 27 & 100,0 & & \\
\hline
\end{tabular}

Fuente: Elaboración propia.

En esta pregunta realizada a docentes de básica elemental de la Escuela Emilio Abad, responden el $77,8 \%$ un sí, manifestando que es positivo introducir las TIC en horas laborables dando como resultado un favorecedor y enriquecido aprendizaje, y el $18,5 \%$ un no rotundo a la propuesta. 


\section{Tabla 4}

Las TIC son muy importantes para la enseñanza en circunstancias de aislamiento

\begin{tabular}{|c|c|c|c|c|c|}
\hline & & Frecuencia & Porcentaje & Porcentaje válido & $\begin{array}{l}\text { Porcentaje } \\
\text { acumulado }\end{array}$ \\
\hline & $\mathrm{Si}$ & 23 & 85,2 & 88,5 & 88,5 \\
\hline \multirow[t]{2}{*}{ Válidos } & No & 3 & 11,1 & 11,5 & 100,0 \\
\hline & Total & 26 & 96,3 & 100,0 & \\
\hline Perdidos & Sistema & 1 & 3,7 & & \\
\hline \multicolumn{2}{|c|}{ Total } & 27 & 100,0 & & \\
\hline
\end{tabular}

Fuente: Elaboración propia.

En lo referente a los resultados conjuntos de la muestra, debo indicar que el $85,2 \%$ de los sujetos dan una valoración positiva, mientras que el $11,1 \%$ califican con una valoración negativa la muestra.

\section{Tabla 5}

Conocimiento adquirido de los alumnos implementando las TIC, como los considera.

\begin{tabular}{|c|c|c|c|c|c|}
\hline & & Frecuencia & Porcentaje & Porcentaje válido & $\begin{array}{l}\text { Porcentaje } \\
\text { acumulado }\end{array}$ \\
\hline \multirow{4}{*}{ Válidos } & Excelente & 12 & 44,4 & 46,2 & 46,2 \\
\hline & Regular & 11 & 40,7 & 42,3 & 88,5 \\
\hline & Malo & 3 & 11,1 & 11,5 & 100,0 \\
\hline & Total & 26 & 96,3 & 100,0 & \\
\hline \multirow{2}{*}{ Perdidos } & Sistema & 1 & 3,7 & & \\
\hline & Total & 27 & 100,0 & & \\
\hline
\end{tabular}

Fuente: Elaboración propia.

Del análisis realizado con respecto a la alternativa de usar las TIC para desarrollar la lectoescritura un $44 \%$ de los encuestados expresan que el uso de métodos y estrategias nuevas dan como resultado destrezas validas, permitiéndoles a los estudiantes desarrollar aptitudes y conocimientos absolutamente propicios para responder y manifestar inquietudes del medio. 
Revista Arbitrada Interdisciplinaria KOINONIA

Año 2020. Vol V. ํ⒈ Especial Educación

Hecho el depósito de Ley: FA2016000010

ISSN: 2542-3088

FUNDACIÓN KOINONIA (F.K). Santa Ana de Coro. Venezuela.

Cristian Javier Luna-Miranda; Darwin Gabriel García-Herrera; Ana Zulema Castro-Salazar; Juan Carlos Erazo-Álvarez

\section{Tabla 6}

Relación entre aprendizaje significativo e integración progresivamente las TIC en su jornada laboral

\begin{tabular}{|c|c|c|c|c|c|c|}
\hline & & & $\begin{array}{l}\text { Le pare } \\
\text { progresive }\end{array}$ & $\begin{array}{r}\text { ositivo el } \\
\text { nte las TI } \\
\text { laboral }\end{array}$ & $\begin{array}{l}\text { r integrando } \\
\text { en su jornada }\end{array}$ & Total \\
\hline & & & Mucho & Poco & Casi nada & \\
\hline & & Recuento & 15 & 3 & 1 & 19 \\
\hline & Mucho & $\begin{array}{c}\text { Frecuencia } \\
\text { esperada }\end{array}$ & 13,9 & 2,9 & 2,2 & 19,0 \\
\hline Las II tavorecen al & & Recuento & 3 & 1 & 1 & 5 \\
\hline significativo en los & Poco & $\begin{array}{c}\text { Frecuencia } \\
\text { esperada }\end{array}$ & 3,7 & ,8 & 6 & 5,0 \\
\hline & & Recuento & 1 & 0 & 1 & 2 \\
\hline & Nada & $\begin{array}{l}\text { Frecuencia } \\
\text { esperada }\end{array}$ & 1,5 & ,3 & ,2 & 2,0 \\
\hline & & Pruebas c & hi-cuadrad & & & \\
\hline & & & & gl & Sig. asintótica & ilateral) \\
\hline Chi-cuadrado de Pear & & & & 4 & ,373 & \\
\hline Razón de verosimilitu & & & & 4 & ,475 & \\
\hline Asociación lineal por I & eal & & & 1 & , 106 & \\
\hline N de casos válidos & & & & & & \\
\hline
\end{tabular}

a. 8 casillas $(88,9 \%)$ tienen una frecuencia esperada inferior a 5 . La frecuencia mínima esperada es, 23. Fuente: Elaboración propia.

Como el valor de sig. (Valor critico observado) $0,000<5$ se rechaza la hipótesis nula y acepta la hipótesis alternativa, es decir que el Uso alternativo de las TIC en Básica Elemental para la lectoescritura se relaciona significativamente con el progresivo uso y aprovechamiento de lo instruido y experimentado dentro del aula de clase, obteniendo como resultados progresivos, una mayor exposición de efectos buenos y accesibles en los alumnos de EGB. 


\section{PROPUESTA}

es sustancial reflexionar sobre los elementos que se ponen en marcha para armar el proyecto. A veces se definen los objetivos a lograr como si ya se hubieran conseguido. La forma en la que cada organización despliega los recursos y los organiza va a ser importante para el logro de los mismos. Entre ello el mejorar el dominio léxico de la lectura y escritura.

Es fundamental establecer y ejecutar un modelo acorde a las nescidades básicas de los niños, esta propuesta metodológica esta muy entrelasada a las nuevas formas de enseñanaza y entre ellas se aplicara las TIC (Tecnologías de la Información y la Comunicación), metodologica que se presta para mejores resultados provechosos dentro y fuera de un establecimiento educativo, este sistema de apoyo no sólo se centra en el papel fundamental de la formación del profesorado.

Es indudable que el colectivo docente necesita, en primer lugar, un proceso de formación y capacitación que la organización del mismo establece un punto relevante. El docente debe tener constante capacitacion y estar al día en los cambios académicos, y las nuevas metodologías de estudio, tambien es importante estar al tanto del uso de las TIC y las innovaciones en la educación debe ser influencia para los estudiantes, los mismos que necesitan de una organización y entrenamiento tanto de la lectura como se la tecnología. Su configuración, funciones y lugar de hecho, dependen del historial de conocimientos y metodologia adquirida y ejecutada en el aula hacia los alumnos. Por lo mismo, esta propuesta es considerada uno de los puntos clave par el éxito en el propósito de integración de las TIC en los procesos de enseñanza-aprendizaje. La forma en que se organizará este mecanismo también recubre importancia: el papel del experto en contenido, cómo y quién se encargará del diseño de ejecución, del diseño digital, si consideramos la presencia de programadores, o cuál va a ser el papel del coordinador del equipo. Este proceo de aplicación se llevara de la siguiente manera: 


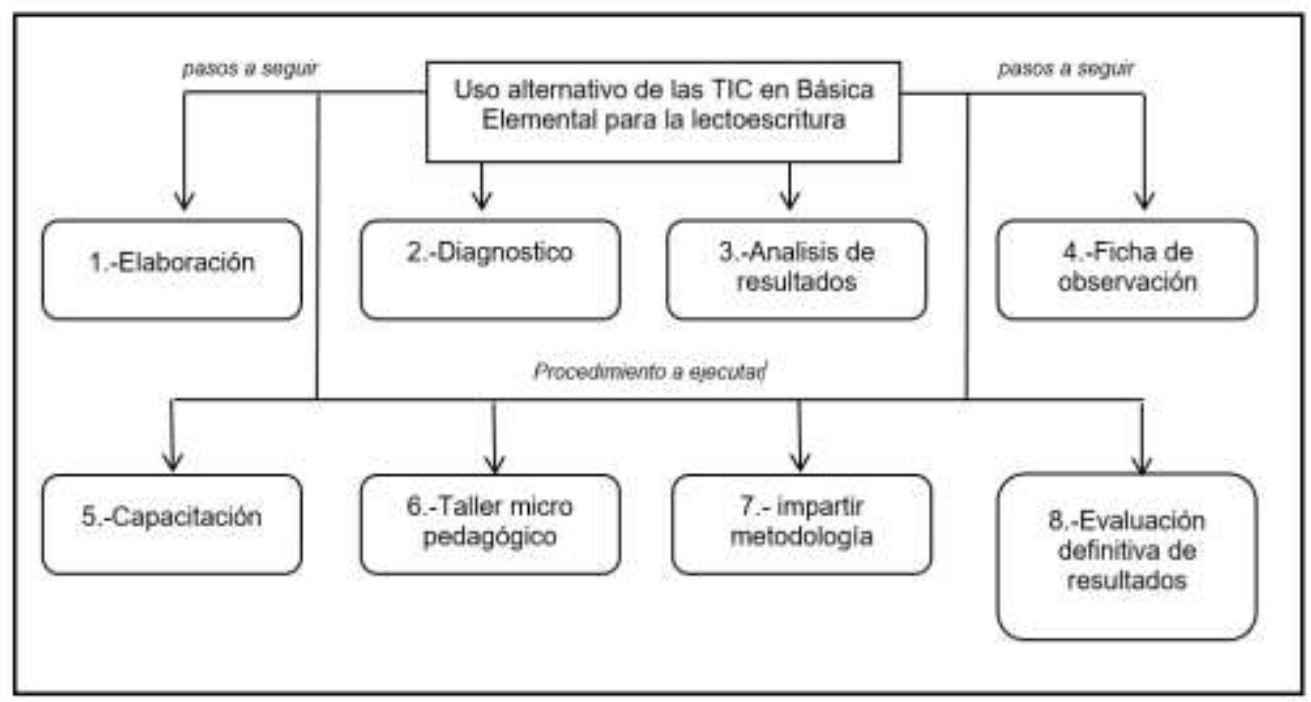

Figura 1. Uso alternativo de las TIC. Fuente: Elaboración propia

1.- Elaboración y planificación del proyecto orientado al desarrollo de la lectoescritura a través del uso de TIC.

2.- Realizar un diagnóstico a los docentes de Educación Básica elemental de la institución educativa, el cual busque tener un panorama claro de la situación y recursos TIC con los que cuentan los docentes y estudiantes.

3.- Analizar los resultados obtenidos en el diagnóstico para proyectar posibles escenarios, problemas y fortalezas para la implementación de la propuesta.

4.- Proyectar una ficha de observación para el alumnado, la cual estárá orientada y se remitira al uso y manipulación de tecnologia por parte de los estudiantes.

5.- Capacitar constantemente al profesorado en torno a las nuevas tendencias metodologicas el uso de las TIC.

6.- Realizar un taller micropedagógico para los estudiantes llevando consigo las distintas formas de adquirir las destrezas de lectura y escritura respetando las secciónes marcadas en el currículo, sin descuidar la utilización de tecnologías para cumplir con este cometido.

Evaluar continuamente el desarrollo del proceso de resultados obtenidos en los mismos, en pro de buscar puntos estratégicos que mejoren la propuesta por ende la lectoescritura 
de los niños.

\section{CONCLUSIONES}

En esta investigación después de analizar los resultados se determinó que el uso de las TIC como es interacción social, imágenes, videos acceso a internet, la creación de contenidos en la web supera los bajos niveles de conocimiento, motivando a la comunidad educativa a implementar todas estas herramientas de contenido de multimedia para su aplicación en los procesos de aprendizaje y hacer más divertido cada actividad que se presenta al transcurso de su vida estudiantil, cabe destacar que en países desarrollados esto ya lo aplican desde periodos atrás, y en países en vía de desarrollo también lo están aplicando pero en menor medida, esto lo hacen para que los niños, niñas y jóvenes permita adaptarse a la nueva sociedad del conocimiento tecnológico.

Con el uso de las TIC, en el ámbito social existe un cambio adecuado y motivador para las diferentes dinámicas cotidianas, en el cual se cambia el rol del docente siendo un mediador, guía de aprendizaje y los estudiantes generan su propio conocimiento y así desarrollan su pensamiento crítico, colaborativo además los docentes deben generar estrategias para la adquisición del conocimiento y generar competencias individuales necesarias para el trabajo individual y colaborativo, para la vida y el mundo en el cual se desarrolla.

El rol del docente en el proceso de aprendizaje de lectoescritura en la institución consiste en precisar una serie de estrategias y criterios de evaluación continua para orientar la producción escrita de textos en situaciones reales, reconociendo la relación de causa efecto entre lectura y escritura al momento de escribir donde al estudiante se le evidencia las competencias adquiridas en el proceso lector, es necesario tomar en cuenta en avance del estudiante al leer, transcribir y producir textos como desarrolla su pensamiento crítico y reflexivo, orientado, no debe observarse como actividad aislante aprender a leer y escribir, al contrario una actividad diaria, motivada e interesante para establecer contacto con el mundo exterior. 
La escuela debe ser diseñada acorde a la edad y propiciar un ambiente acogedor que facilite nuevos aprendizajes. Se debe adecuar un rincón tecnológico en el aula, siendo este necesario que promueva dichos aprendizajes, además es necesario fomentar el trabajo cooperativo en el aula, desde los cuales se administre recursos de espacio, tiempo, actividades y recursos. Por otro lado, el docente es un modelo a seguir, si el aplica el gusto por leer, sus estudiantes van a seguir el modelo, el docente se involucra en este proceso y permite que el estudiante supere obstáculos en el transcurso de su vida estudiantil.

\section{FINANCIAMIENTO}

No monetario.

\section{AGRADECIMIENTO}

A los docentes de Educación Básica Elemental de la Escuela Emilio Abad, por apoyar el desarrollo de este proceso investigativo.

\section{REFERENCIAS CONSULTADAS}

Andersen -Boris Branisa, Lykke E., \& Stefano Canelas. (2016). El ABC del desarrollo en Bolivia. [The ABC of development in Bolivia]. Fundación INESAD. Recuperado de https://n9.cl/6of8

Argudo-Tello, K. J., Erazo-Álvarez, J. C., \& Narváez-Zurita, C. I. (2019). Evaluación de Control Interno en Riesgos Estratégicos para la Dirección de Planificación de la Universidad de Cuenca. [Evaluation of Internal Control in Strategic Risks for the Planning Direction of the University of Cuenca]. Revista Arbitrada Interdisciplinaria Koinonía, 67-96. http://dx.doi.org/10.35381/r.k.v4i1.372

Bajaña Delgado, Rosa Elizabeth. (2017). Rol de los espacios de aprendizaje para fomentar los hábitos de lectura en los estudiantes de la Unidad Educativa Isabel la Católica. [Role of learning spaces to promote reading habits in the students of the Isabel la Católica Educational Unit]. Recuperado de https://n9.cl/azhqk 
Bazán-Ramírez, Aldo, Eduardo Hernández-Padilla, César A. Hernández-Rodríguez, \& Eneida Ochoa-Avila. (2020). Relaciones entre contexto de procedencia y logro académico al terminar la primaria. [Relations between context of origin and academic achievement at the end of primary school]. Revista Electrónica de Investigación Educativa 22(1):1. Recuperado de https://n9.cl/qth74

Borregales, L. (2020). Génesis y evolución socio-histórica de la macro categoría cultura escrita. [Genesis and socio-historical evolution of the macro category written

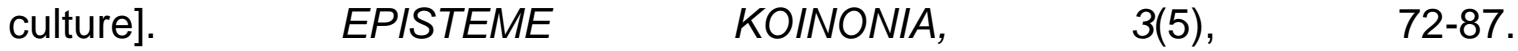
http://dx.doi.org/10.35381/e.k.v3i5.529

Coll, César. (2008). Aprender y enseñar con las TIC: Expectativas, realidad y potencialidades. [Learning and teaching with ICT: Expectations, reality and potential]. Recuperado de https://n9.cl/g48rz

Chacha-Supe, M., \& Rosero-Morales, E. (2020). Procesos iniciales de enseñanza aprendizaje de la lectoescritura en los estudiantes del nivel de preparatoria. [Initial teaching-learning processes of literacy in high school students]. Revista Arbitrada Interdisciplinaria Koinonía, 5(9), 311-336. http://dx.doi.org/10.35381/r.k.v5i9.650

Dovale, Y. (2018). Las habilidades resuntivas de ensayos académicos expositivos en estudiantes de la Universidad Nacional Experimental Francisco de Miranda. [The residual skills of expository academic essays in students of the Francisco de Miranda National Experimental University]. EPISTEME KOINONIA, 1(1), 18-34. http://dx.doi.org/10.35381/e.k.v1i1.488

Dezcallar, Teresa, Mercè Clariana, Ramón Cladellas, Mar Badia, \& Concepción Gotzens. (2014). La lectura por placer: su incidencia en el rendimiento académico, las horas de televisión y las horas de videojuegos. [The pleasure of reading: its impact on academic achievement, on TV-watching hours and video games-playing hours]. Ocnos, 107-16. Recuperado de https://n9.cl/shlb3

Duart, Josep M. (2012). Full Issue. [Problema completo]. RUSC. Universities and Knowledge Society Journal 9(1):1. Recuperado de https://n9.cl/v9e6

Escotto, Eduardo. (2014). Intervención de la lectoescritura en una niña con dislexia. [Literacy intervention in a girl with dyslexia]. Pensamiento Psicológico. Recuperado de https://n9.cl/zmbj 
Lazo-Torres, N. S., Erazo-Álvarez, J. C., \& Narváez-Zurita, C. I. (2019). El Balanced Scorecard como herramienta de control interno en el sector Manufacturero [The Balanced Scorecard as an internal control tool in the Manufacturing sector]. Revista Arbitrada Interdisciplinaria Koinonía, 125-152. doi:http://dx.doi.org/10.35381/r.k.v4i1.374

Luz, Cacheiro María. (2018). Educación y tecnología: estrategias didácticas para la integración de las TIC. [Education and technology: didactic strategies for the integration of ICT]. Editorial UNED. Recuperado de https://n9.cl/bttdn

Magán-Hervás, Alexandra, \& Felipe Gértrudix-Barrio. (2017). Influencia de las actividades audio-musicales en la adquisición de la lectoescritura en niños y niñas de cinco años. [Influence of audio-musical activities on the acquisition of literacy in boys and girls of five years]. Revista Electrónica Educare 21(1):288-309. Doi: http://dx.doi.org/10.15359/ree.21-1.15

Mendieta-Ortega, M. P., Erazo-Álvarez, J. C., \& Narváez-Zurita, C. I. (2020). Gestión por competencias: herramienta clave para el rendimiento laboral del talento humano del sector hospitalario [Management by competences: key tool for the labor performance of the human talent of the hospital sector]. Revista Arbitrada Interdisciplinaria Koinonía, 287-312. doi:http://dx.doi.org/10.35381/r.k.v5i10.696

Navarro, Federico, Natalia Ávila Reyes, Mónica Tapia-Ladino, Vera L. L. Cristóbal, María Ester W. Moritz, Elizabeth Narváez Cardona, \& Charles Bazerman. (2016). Panorama histórico y contrastivo de los estudios sobre lectura y escritura en educación superior publicados en América Latina. [Historical and contrastive overview of studies on reading and writing in higher education published in Latin America]. Revista Signos 49:100-126. Doi: http://dx.doi.org/10.4067/S0718$\underline{09342016000400006}$

Organización Panamericana de la Salud. (2020). Especificaciones Técnicas de dispositivos médicos para la gestión de casos de Covid-19 en los servicios de salud. [Technical specifications of medical devices for case management of Covid19 in health services]. Organización Mundial de La Salud 1-8. Recuperado de https://n9.cl/48jzv

Palos Toscano, María Úrsula, María Luisa Ávalos Latorre, Francisco Flores Escobar, \& Roberto Montes Delgado. (2017). Creencias de madres y docentes sobre el aprendizaje de la lectoescritura en educación preescolar. [Beliefs of mothers and teachers about learning to read and write in preschool education]. Actualidades Investigativas En Educación 17(3):168-89. Doi: http://dx.doi.org/10.15517/aie.v17i3.29066 
Porta, María Elsa, \& Hilda Anglat. (2011). Identificando predictores del aprendizaje lingüístico inicial: efecto de factores cognitivos, lingüísticos y ambientales. [Identifying predictors of initial language learning: effect of cognitive, linguistic and environmental factors]. Psico/Pedagógica. Recuperado de https://n9.cl/uzx9

Prado-Huante, H. (1949). Educación y Psicología. [Education and Psychology]. Psiquis 4(6):361-64. Recuperado de https://n9.cl/igev

Suárez, Ana, Claudia Pérez, María Vergara, \& Alférez Víctor. (2015). Desarrollo de la lectoescritura mediante TIC y recursos educativos abiertos. [Development of literacy through ICT and open educational resources]. Apertura, 1-7. Recuperado de https://n9.cl/b5pe7

Tamayo Lorenzo, Susana. (2017). La dislexia y las dificultades en la adquisición de la lectoescritura. [Dyslexia and difficulties in acquiring literacy]. Profesorado, 423432. Recuperado de https://n9.cl/0jn8

Tangarife, Diego, Segundo Blanco, \& Gloria Diaz. (2016). Tecnologías y metodologías aplicadas en la enseñanza de la lectoescritura a personas con síndrome de Down. [Tecnologies and methodologies applied in teaching literacy to people with Down syndrome]. Digital Education Review, 265-283. Recuperado de https://n9.cl/wtrq

Toro, Josep, \& Montserrat Cervera. (2015). T.A.L.E.: Test de análisis de lectoescritura. [Literacy test]. Recuperado de https://n9.cl/pwsmw

Vélez-Loor, M., Vallejo-Valdivieso, P., \& Moya-Martínez, M. (2020). Recursos didácticos virtuales en proyectos de ciencias naturales en período de confinamiento por COVID-19. [Virtual teaching resources in natural science projects in confinement period by COVID-19]. EPISTEME KOINONIA, 3(5), 183-201. http://dx.doi.org/10.35381/e.k.v3i5.760 